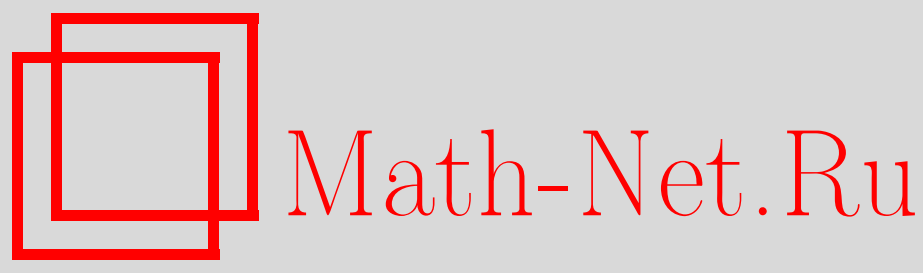

О. В. Держко, Т. Е. Крохмальский, Й. Рихтер, Квантовый антиферромагнетик Гейзенберга на низкоразмерных фрустрированных решетках, ТМФ, 2011, том 168, номер 3, 441-452

DOI: https://doi.org/10.4213/tmf6692

Использование Общероссийского математического портала Math-Net.Ru подразумевает, что вы прочитали и согласны с пользовательским соглашением http://www . mathnet.ru/rus/agreement

Параметры загрузки:

IP : 54.198 .67 .100

26 апреля 2023 г., 16:01:19

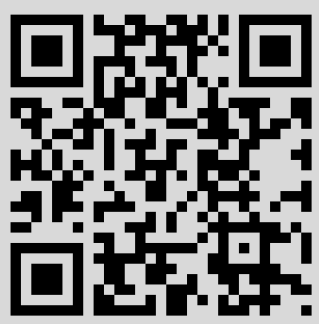




\title{
ФИЗИКА
}

Том 168, № 3

сентябрь, 2011

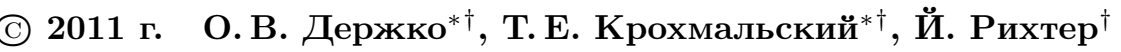

\section{КВАНТОВЫЙ АНТИФЕРРОМАГНЕТИК ГЕЙЗЕНБЕРГА НА НИЗКОРАЗМЕРНЫХ ФРУСТРИРОВАННЫХ РЕШЕТКАХ}

\begin{abstract}
Иссследованы низкотемпературные процессы намагничивания для двух фрустрированных квантовых антиферромагнетиков Гейзенберга с использованием модели решеточного газа. Возникающие дискретные степени свободы предполагают тесную связь фрустрированного квантового антиферромагнетика Гейзенберга с классическим решеточным газом с конечным отталкиванием ближайших соседей или, эквивалентно, с антиферромагнетиком Изинга в однородном магнитном поле. С учетом этой связи получены аналитические результаты для термодинамически больших систем в одномерном случае. В двумерном случае выполнено численное моделирование по классическому методу Монте-Карло для систем размером вплоть до $100 \times 100$ узлов.
\end{abstract}

Ключевые слова: квантовый антиферромагнетик Гейзенберга, фрустрированные решетки, процесс намагничивания.

\section{1. ВВЕДЕНИЕ}

Экспериментальные и теоретические исследования процессов намагничивания являются актуальной проблемой современной физики конденсированных систем. Хотя здравый смысл предполагает, что намагниченность $M$ монотонно возрастает, достигая насыщения, с возрастанием приложенного поля $h$, экспериментальные наблюдения для многих материалов часто демонстрируют нетривиальную зависимость $M$ от $h$, содержащую плато и скачки при низких температурах (см., например, работы [1]). Этот тип поведения может быть вызван конкуренцией взаимодействий, которая имеет место во фрустрированных квантовых системах. Значительный интерес в последние десятилетия вызывает теоретическое описание процессов намагничивания во фрустрированных квантовых спиновых системах, в частности предсказания поведения кривых низкотемпературной намагниченности термодинамически больших систем [1].

* Институт физики конденсированных систем НАН Украины, Львов, Украина

${ }^{\dagger}$ Львовский национальный университет им. И. Франко, Львов, Украина.

E-mail: derzhko@icmp.lviv.ua

${ }^{\ddagger}$ Institit fur Theoretische Physik, Otto-von-Guericke-Universität Magdeburg, Magdeburg, Deutchland. E-mail: Johannes.Richter@physik.uni-magdeburg.de 
С другой стороны, для квантового антиферромагнетика Гейзенберга на определенных фрустрированных решетках недавно была предложена концепция независимых локализованных магнонных состояний [2]. Эта концепция с успехом использовалась для описания магнитотермодинамики таких систем [3]. Независимые локализованные магнонные состояния являются основными состояниями в сильных магнитных полях, и поэтому картина независимых локализованных магнонов (или представление с помощью модели жестких ко́ров) в режиме сильных полей и низких температур дает адекватное описание. Более того, совсем недавно описание при помощи независимых локализованных магнонов было улучшено: для некоторых решеток [4] оказалось возможным включить в рассмотрение также и низкоэнергетические возбужденные состояния (взаимодействующие локализованные магнонные состояния) [5]. Эти низкоэнергетические степени свободы некоторых фрустрированных квантовых спиновых систем могут быть также описаны с помощью подходящей теории решеточного газа. В нашей работе [5] были представлены некоторые результаты для термодинамических величин (в основном касающиеся температурных зависимостей теплоемкости, прыжковой (подрешеточной) восприимчивости и энтропии). Цель настоящей работы - изучение процессов намагничивания при низких температурах, которые до сих пор не исследовались, с помощью теории решеточного газа. Мы продемонстрируем, что такое модельное описание рассматриваемых фрустрированных квантовых магнетиков очень точно воспроизводит низкотемпературные величины.

Статья организована следующим образом. В разделе 2 определены квантовые спиновые модели и объяснено возникновение описания с помощью решеточного газа. В разделе 3 вычисляется намагниченность фрустрированных квантовых спиновых систем отдельно в одномерном и двумерном случаях. Там же представлены результаты для термодинамически больших систем, полученные с использованием метода матрицы переноса (одномерный случай) и численным моделированием по классическому методу Монте-Карло (двумерный случай). Далее в рамках описания с помощью решеточного газа детально обсуждены низкотемпературные однородные намагниченность и восприимчивость. Наконец, в разделе 4 подводятся итоги.

\section{2. ФРУСТРИРОВАННЫЕ КВАНТОВЫЕ СПИНОВЫЕ СИСТЕМЫ. ОПИСАНИЕ С ПОМОЩЬЮ РЕШЕТОЧНОГО ГАЗА}

Мы рассматриваем $N=2 \mathcal{N}$ квантовых спинов $1 / 2$, расположенных на двух решетках, которые показаны на рис. 1 (см. также работы [6], [7]). Гамильтониан модели имеет вид

$$
H=\sum_{(p q)} J_{p q} \mathbf{s}_{p} \cdot \mathbf{s}_{q}-h S^{z},
$$

где суммирование ведется по связям, соединяющим соседние узлы на решетке; $J_{p q}>0$ - антиферромагнитное взаимодействие между узлами $p$ и $q ; J_{p q}$ принимает два значения: $J_{2}$ для вертикальных связей и $J_{1}$ для горизонтальных и диагональных связей; $h \geqslant 0$ - внешнее магнитное поле; $S^{z}=\sum_{p=1}^{N} s_{p}^{z}-z$-компонента полного спина системы. В нашем исследовании мы налагаем периодические граничные условия и обычно полагаем $J_{1}=1$ для фиксирования шкалы энергий.

Целесообразно ввести полный спин на вертикальной связи $\mathbf{t}_{m}=\mathbf{s}_{m, 1}+\mathbf{s}_{m, 2}$. Здесь $m=1, \ldots, \mathcal{N}$ нумерует вертикальные связи и пробегает по узлам базовой решетки, т. е. простой цепочки в случае фрустрированной двуногой спиновой лестницы или 


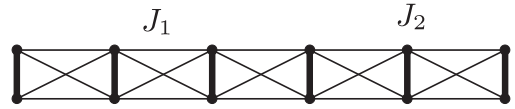

a

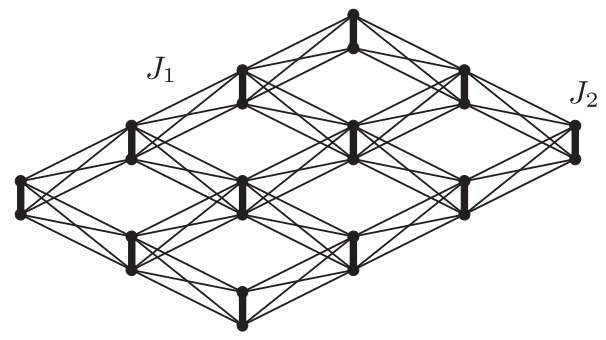

б

Рис. 1. Две решетки, рассматриваемые в настоящей статье: фрустрированная двуногая спиновая лестница (а) и фрустрированный двойной спиновый слой (б).

квадратной решетки в случае фрустрированного двойного спинового слоя. Тогда гамильтониан (2.1) принимает вид

$$
H=\sum_{m}\left[\frac{J_{2}}{2}\left(\mathbf{t}_{m}^{2}-\frac{3}{2}\right)-h t_{m}^{z}\right]+J_{1} \sum_{(m l)} \mathbf{t}_{m} \cdot \mathbf{t}_{l},
$$

где вторая сумма пробегает по связям между ближайшими соседями на базовой решетке (простой цепочке или квадратной решетке). Из уравнения (2.2) очевидно, что полный спин $t_{m}$ на каждой вертикальной связи $m=1, \ldots, \mathcal{N}, \mathbf{t}_{m}^{2}=t_{m}\left(t_{m}+1\right)$, является хорошим квантовым числом. Следовательно, гамильтониан (2.2) зависит от набора квантовых чисел $\left\{t_{m}\right\}, t_{m}=0,1$. Это позволяет изучить свойства модели более детально. В частности, может быть построено точно подмножество $2^{\mathcal{N}}=(1.41 \ldots)^{N}$ низколежащих собственных состояний из полного множества $2^{N}$ собственных состояний [5], [6]. Более того, их вклад в термодинамику может быть вычислен с помощью классической модели решеточного газа [5].

Начнем с рассмотрения собственных состояний, которые более детально будут изучены далее. Сначала рассмотрим состояния, содержащие $n$ синглетов на вертикальных связях $m_{1}, \ldots, m_{n}$ (т. е. $\left.\mathbf{t}_{m_{i}}^{2}=0\right)$ и $\mathcal{N}-n$ полностью поляризированных триплетов $\left(\mathbf{t}_{m}^{2}=2, t_{m}^{z}=1\right)$ на остальных вертикальных связях. Наложим правило жесткого кора, т. е. запретим заполнение синглетами соседних вертикальных связей. Следуя обозначениям, введенным в работах [2]-[4], назовем эти состояния "независимыми локализованными магнонными состояниями", а синглет на вертикальной связи - “локализованным магноном". Энергия этих независимых локализованных магнонных состояний равна $E_{n}^{\mathrm{lm}}=E_{\mathrm{FM}}-n \epsilon_{1}$, где $E_{\mathrm{FM}}=\mathcal{N} J_{1}+\mathcal{N} J_{2} / 4$, $\epsilon_{1}=J_{2}+2 J_{1}=h_{1}$ в одномерном случае и $E_{\mathrm{FM}}=2 \mathcal{N} J_{1}+\mathcal{N} J_{2} / 4, \epsilon_{1}=J_{2}+4 J_{1}=h_{1}$ в двумерном случае. Кратность вырождения независимых локализованных магнонных состояний $g_{\mathcal{N}}(n)$ равна канонической статистической сумме $\mathcal{Z}_{\mathrm{hc}}(n, \mathcal{N}) n$ жестких объектов (ко́ров) (жестких димеров или жестких квадратов) на базовой решетке (простой цепочке или квадратной решетке) из $\mathcal{N}$ узлов. Важно заметить, что независимые локализованные магнонные состояния являются основными состояниями в подпространствах с $S^{z}=\mathcal{N}-1, \ldots, \mathcal{N} / 2$, если $J_{2} / J_{1} \geqslant 2$ (одномерный случай) или $J_{2} / J_{1} \geqslant 4$ (двумерный случай) (см. работу [8]), а также то, что эти состояния линейно независимы [9]. 
Далее мы ослабляем правило жесткого кора, т. е. разрешаем локализованным магнонам (синглетам на вертикальных связях) быть ближайшими соседями. Согласно работе [5] назовем эти состояния "взаимодействующими локализованными магнонными состояниями". Каждая пара соседствующих локализованных магнонов увеличивает энергию на $J_{1}$. В результате для заданного $S^{z}=\mathcal{N}-n, n=1, \ldots, \mathcal{N} / 2$, энергии этих взаимодействующих локализованных магнонных возбужденных состояний становятся равными $E_{n}^{\nu}=E_{n}^{\operatorname{lm}}+\nu J_{1}$, где $\nu$ - число пар соседствующих локализованных магнонов (см. также работу [5]). Взаимодействующие локализованные магнонные состояния являются низколежащими возбужденными состояниями для $S^{z}=\mathcal{N}-n, n=1, \ldots, \mathcal{N} / 2$, в режиме сильного взаимодействия, т. е. когда отношение $J_{2} / J_{1}$ достаточно велико. Точнее, из данных точной диагонализации для конечных систем мы установили, что режим сильного взаимодействия имеет место, когда $J_{2}>J_{2}^{\mathrm{c}}$ при $J_{2}^{\mathrm{c}} / J_{1} \approx 3.00$ (одномерный случай) и $J_{2}^{\mathrm{c}} / J_{1} \approx 4.65$ (двумерный случай) [5].

Для меньших значений намагниченности $S^{z}=\mathcal{N} / 2-r, r=1, \ldots, \mathcal{N} / 2$, когда независимые локализованные магнонные состояния уже не существуют, класс взаимодействующих локализованных магнонных состояний в режиме сильного взаимодействия содержит как множество основных состояний, так и низколежащие возбужденные состояния. Множество основных состояний построено из $n=\mathcal{N} / 2+r$, $r=1, \ldots, \mathcal{N} / 2$, локализованных магнонов, где, например, $\mathcal{N} / 2$ магнонов полностью заполняют одну подрешетку базовой решетки (простой цепочки или квадратной решетки), а остальные $r$ локализованных магнонов находятся на другой подрешетке. Энергия этого состояния равна $E_{\mathcal{N} / 2+r}=-\mathcal{N} J_{2} / 4-r J_{2}, J_{2}=h_{2}$. Низколежащие возбужденные состояния строятся из основного состояния перегруппировкой локализованных магнонов, чтобы увеличить число соседствующих магнонов. Тогда каждая новая пара соседних локализованных магнонов увеличивает энергию на $J_{1}$.

Заметим, что взаимодействующие локализованные магнонные состояния можно визуализировать как частично перекрывающиеся жесткие объекты (в отличие от независимых локализованных магнонных состояний, которые можно визуализировать как неперекрывающиеся жесткие объекты). Хотя кратность вырождения взаимодействующих локализованных магнонных состояний может быть также вычислена в терминах канонической статистической суммы системы жестких объектов, нужный вклад независимых и взаимодействующих локализованных магнонных состояний в статистическую сумму $Z(T, h, N)$ квантовой спиновой системы (2.1) удобно учитывается в рамках модели решеточного газа классических частиц с конечным отталкиванием ближайших соседей $V=J_{1}$ (см. работу [5]).

В режиме сильного взаимодействия, когда сконструированные независимые и взаимодействующие локализованные магнонные состояния доминируют в статистической сумме $Z(T, h, N)$ квантовой спиновой системы (2.1) при низких температурах, для $Z(T, h, N)$ можно написать [5]

$$
\begin{aligned}
Z(T, h, N) & \approx Z_{\mathrm{LM}}(T, h, N)= \\
& =\sum_{n_{1}=0,1} \cdots \sum_{n_{\mathcal{N}}=0,1} e^{-\left(E_{\mathrm{FM}}-h \mathcal{N}+\left(h-h_{1}\right) \sum_{m} n_{m}+J_{1} \sum_{(m l)} n_{m} n_{l}\right) / T}= \\
& =e^{-\left(E_{\mathrm{FM}}-h \mathcal{N}\right) / T} \Xi_{\mathrm{lg}}(T, \mu, \mathcal{N}),
\end{aligned}
$$

где $\Xi_{\lg }(T, \mu, \mathcal{N})=\sum_{n_{1}=0,1} \cdots \sum_{n_{\mathcal{N}}=0,1} e^{-\mathcal{H}\left(\left\{n_{m}\right\}\right) / T}-$ большая каноническая статистическая сумма решеточного газа с гамильтонианом $\mathcal{H}\left(\left\{n_{m}\right\}\right)=-\mu \sum_{m} n_{m}+$ 
$J_{1} \sum_{(m l)} n_{m} n_{l}, \mu=h_{1}-h$. Введя узловые спиновые переменные $\sigma_{m}= \pm 1$ согласно соотношениям $\sigma_{m}=2 n_{m}-1$ и $n_{m}=\left(1+\sigma_{m}\right) / 2$, приходим к гамильтониану антиферромагнитной модели Изинга в однородном магнитном поле.

В работе [5] мы выполнили обширные исследования соотношения между фрустрированной квантовой спиновой моделью (2.1) и классической моделью решеточного газа с гамильтонианом $\mathcal{H}\left(\left\{n_{m}\right\}\right)$ для конечных систем (до $N=32$ ), чтобы выяснить, до какой степени последняя модель может воспроизвести термодинамические свойства предыдущей модели в режиме сильного взаимодействия. В частности, мы проверили энергии и кратности вырождения низколежащих состояний для $S^{z}=N / 2, \ldots, 0$, сравнили полевые и температурные зависимости различных термодинамических величин, оценили температурную область, в которой описание с помощью решеточного газа находится в блестящем согласии с данными точной диагонализации. Так, для фрустрированной двуногой спиновой лестницы с $J_{1}=1$, $J_{2}=4$ (характеристические поля равны $h_{2}=4$ и $h_{1}=6$ ) и для фрустрированного двойного спинового слоя с $J_{1}=1, J_{2}=5$ (характеристические поля равны $h_{2}=5$ и $\left.h_{1}=9\right)$ мы обнаружили, что картина решеточного газа работает великолепно по крайней мере до $T=0.5$ (и даже до $T=1$, если $J_{2}$ имеет большее значение, $\left.J_{2}=10\right)$. Чтобы поддержать этот вывод, в настоящей работе мы выполнили сравнение данных точной диагонализации и предсказаний теории решеточного газа для теплоемкости конечной системы из $N=16$ узлов. Эти результаты показаны на фазовых диаграммах на рис. 2. Мы нашли, что ниже пунктирных линий на фазовых диаграммах на рис. 2 данные о теплоемкости в полной спиновой модели и в рамках описания с помощью решеточного газа совпадают между собой с точностью до $5 \%$, $2 \%$ и $1 \%$ (сверху вниз).

Рассматриваемые фрустирированные квантовые спиновые системы обнаруживают интересное низкотемпературное поведение, если магнитное поле $h$ находится в пределах между $h_{2}$ и $h_{1}$, что связано с упорядочением локализованных магнонов на простой цепочке или квадратной решетке. Обе эти решетки состоят из двух частей (являются двухподрешеточными), т. е. состоят из двух подрешеток $A$ и $B$, и любые соседние узлы всегда принадлежат подрешетке $A$ и подрешетке $B$. В двумерном случае заполнение подрешеток локализованными магнонами реализуется как фазовый переход порядок-беспорядок, который имеет прямую аналогию с фазовым переходом в антиферромагнетике Изинга на квадратной решетке в однородном магнитном поле и принадлежит к классу универсальности двумерной модели Изинга. Имеется много исследований антиферромагнитной модели Изинга на квадратной решетке в однородном магнитном поле (см., например, работы [10]-[13]), и мы можем позаимствовать существующие результаты для исследования двумерного квантового антиферромагнетика Гейзенберга на фрустрированном двойном спиновом слое. В частости, обсуждалась фазовая диаграмма в полуплоскости "магнитное поле-температура" антиферромагнетика Изинга на квадратной решетке в однородном магнитном поле и была вычислена в различных приближениях линия критической температуры $T_{\mathrm{c}}(h)$, отделяющая антиферромагнитно-упорядоченную фазу и неупорядоченную фазу. Эта фазовая диаграмма в контексте фрустрированного двойного спинового слоя была воспроизведена классическим методом Монте-Карло [5] (см. рис. 2б). При пересечении критической линии термодинамические величины для фрустрированного двойного спинового слоя проявляют сингулярности: теплоемкость имеет логарифмическую сингулярность, шахматная (подрешеточная) намагниченность (которая может играть роль параметра порядка) затухает в ("ан- 
446

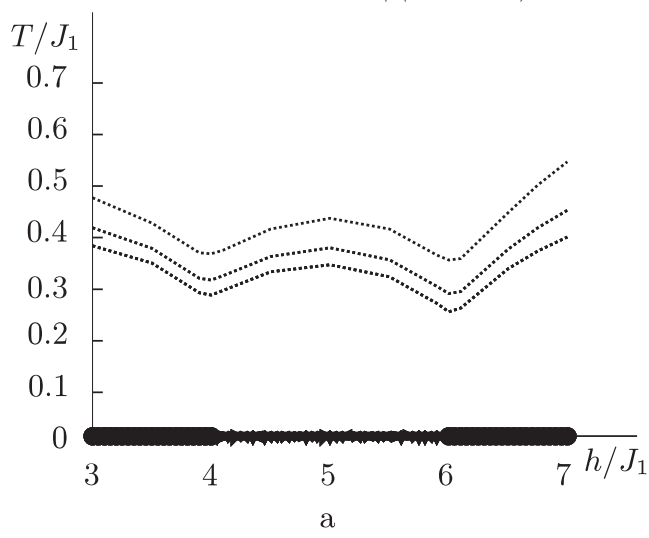

О.В. ДЕРЖКО, Т.Е. КРОХМАЛЬСКИЙ, Й. РИХТЕР

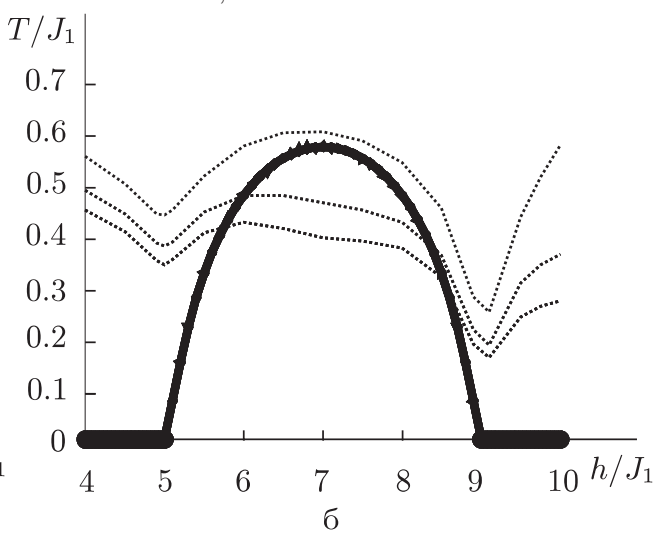

Рис. 2. Фазовые диаграммы, следующие из описания с помощью решеточного газа, для $s=1 / 2$ антиферромагнетика Гейзенберга в магнитном поле на фрустрированной двуногой спиновой лестнице с $J_{1}=1, J_{2}=4\left(h_{2}=4\right.$, $\left.h_{1}=6\right)\left(\right.$ ) и на фрустрированном двойном спиновом слое с $J_{1}=1, J_{2}=5$ $\left(h_{2}=5, h_{1}=9\right)($ б). Заполнение в шахматном порядке вертикальных связей локализованными магнонами (некий "антиферромагнитный" дальний порядок) имеет место вдоль линии между $h_{2}$ и $h_{1}$ при $T=0$ (спиновая лестница) или ниже критической линии $T_{\mathrm{c}}(h)$ с начальной и конечной точками при $h_{2}$, $T=0$ и $h_{1}, T=0$ (двойной спиновый слой), в то время как однородное заполнение вертикальных связей (“ферромагнитный” дальний порядок) имеет место вдоль линий $h<h_{2}$ при $T=0$ и $h_{1}<h$ при $T=0$. Остальная часть фазовых диаграмм соответствует неупорядоченной фазе. Показаны линии, ниже которых данные точной диагонализации и предсказания теории решеточного газа для теплоемкости конечной системы из $N=16$ узлов совпадают между собой с точностью до $5 \%, 2 \%$, и $1 \%$ (пунктирные линии сверху вниз).

тиферромагнитно") упорядоченной фазе с показателем $\beta=1 / 8$, шахматная (подрешеточная) восприимчивость разбегается с показателем $\gamma=7 / 4$.

Используя разработанную картину решеточного газа, в настоящей работе мы вычислим однородную намагниченность $M(T, h, N)$ и однородную восприимчивость $\chi(T, h, N)=\partial M(T, h, N) / \partial h$, которые обе важны и могут быть легко получены в экспериментальных исследованиях.

\section{3. ОДНОРОДНЫЕ НАМАГНИЧЕННОСТЬ И ВОСПРИИМЧИВОСТЬ}

Мы будем использовать уравнение (2.3) для вычисления $M(T, h, N)$ и $\chi(T, h, N)$ для $h \geqslant 0$ при низких температурах $T$ для фрустрированного квантового антиферромагнетика Гейзенберга (2.1) на рассматриваемых решетках. Применяя стандартные формулы для $M$ и $\chi$, получаем

$$
\begin{aligned}
M(T, h, N) & =-\frac{\partial}{\partial h}[-T \ln Z(T, h, N)]=\mathcal{N}-\bar{n}, \quad \bar{n}=T \frac{\partial \ln \Xi_{\lg }(T, \mu, \mathcal{N})}{\partial \mu}, \\
\chi(T, h, N) & =\frac{\partial M(T, h, N)}{\partial h}=\frac{\partial \bar{n}}{\partial \mu}=\frac{1}{T}\left(\overline{n^{2}}-\bar{n}^{2}\right),
\end{aligned}
$$



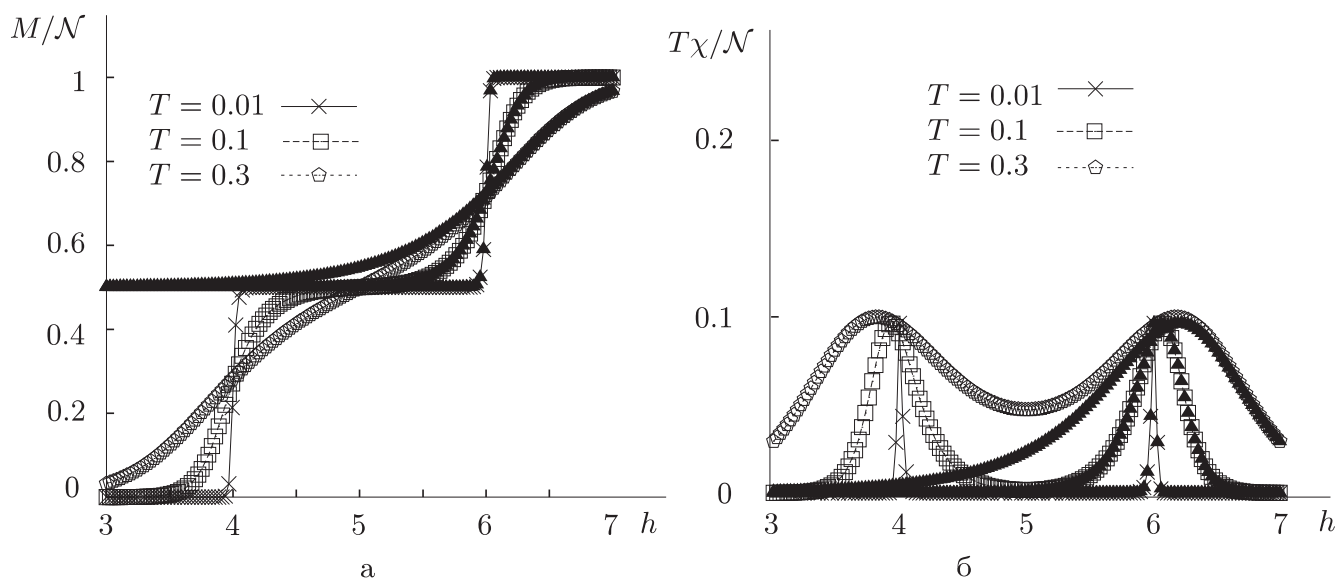

Рис. 3. Полученные методом матрицы переноса результаты для фрустрированной двуногой спиновой лестницы (2.1) с $J_{1}=1, J_{2}=4, \mathcal{N} \rightarrow \infty$ : зависимости $M / \mathcal{N}$ от $h($ а) и $T \chi / \mathcal{N}$ от $h(б)$ при различных температурах $T=0.01,0.1,0.3$. Заполненные треугольники соответствуют предсказаниям модели жестких димеров, которые справедливы только в окрестности $h_{1}$ при очень низких температурах.

где $\overline{(\ldots)}=\sum_{n_{1}=0,1} \cdots \sum_{n_{\mathcal{N}}=0,1}(\ldots) e^{-\mathcal{H}\left(\left\{n_{m}\right\}\right) / T} / \Xi_{\lg }(T, \mu, \mathcal{N})$ обозначает большое каноническое усреднение для модели классического решеточного газа, а $n=\sum_{m=1}^{\mathcal{N}} n_{m}$.

3.1. Фрустрированная двуногая спиновая лестница. В одномерном случае мы используем метод матрицы переноса [14], чтобы получить в явной форме $\Xi_{\lg }(T, \mu, \mathcal{N})$ и, следовательно, $\bar{n}$ и $\partial \bar{n} / \partial \mu$ (см. уравнения (3.1)). После довольно длинных, но несложных вычислений мы приходим к следующему конечному результату в термодинамическом пределе $\mathcal{N} \rightarrow \infty$ :

$$
\begin{gathered}
\frac{M(T, h, N)}{\mathcal{N}}=1-\frac{c_{+}}{\lambda_{+}}, \\
\frac{T \chi(T, h, N)}{\mathcal{N}}=\frac{d_{+}}{\lambda_{+}}-\frac{c_{+}^{2}}{\lambda_{+}^{2}}, \\
\lambda_{+}=\frac{1+w+\sqrt{(1-w)^{2}+4 z}}{2}, \\
c_{+}=\frac{2 z-w(1-w)}{2 \sqrt{(1-w)^{2}+4 z}}+\frac{w}{2}, \\
d_{+}=\frac{[2 z-w(1-w)]^{2}}{2 \sqrt{(1-w)^{2}+4 z}}-\frac{w}{2\left[(1-w)^{2}+4 z\right]^{3 / 2}}, \frac{w}{2},
\end{gathered}
$$

где использованы обозначения $z=e^{\left(h_{1}-h\right) / T}, h_{1}=J_{2}+2 J_{1}, w=z e^{-J_{1} / T}$.

Некоторые результаты для зависимостей $M$ от $h$ и $\chi$ от $h$ при низких температурах, полученные на основе уравнений (3.2), представлены на рис. 3 и обсуждаются в п. 3.3 . 

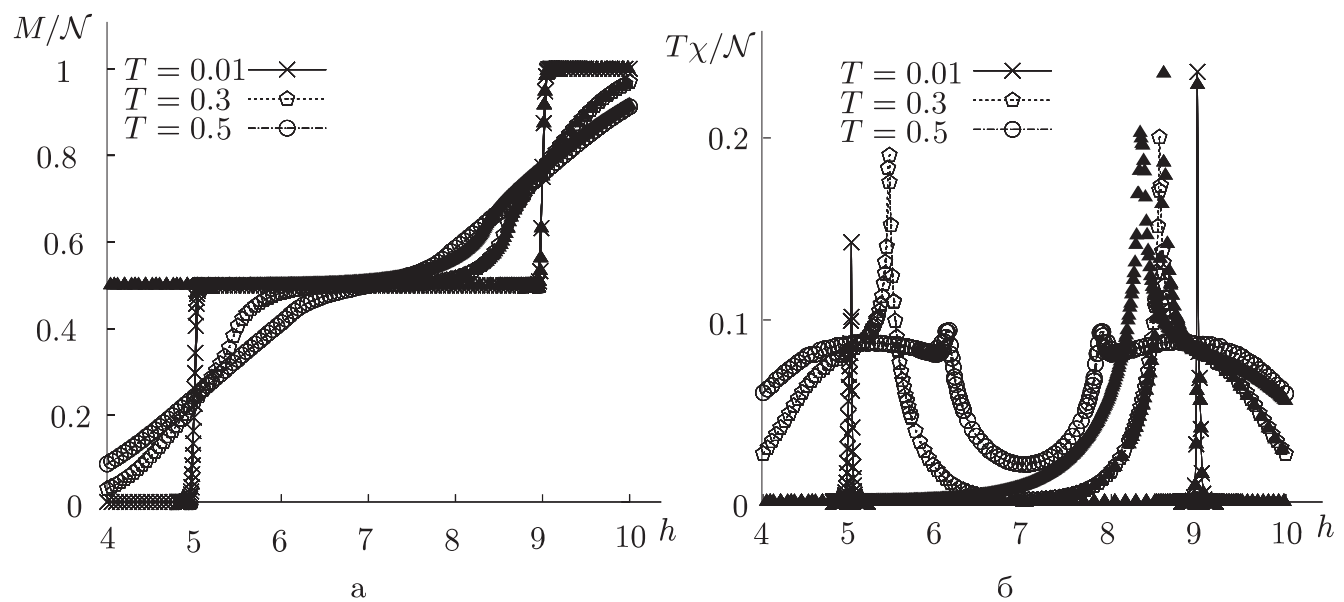

Рис. 4. Результаты, полученные по классическому методу Монте-Карло для фрустрированного двойного спинового слоя $(2.1)$ с $J_{1}=1, J_{2}=5, \mathcal{N}=50 \times 50$ и $\mathcal{N}=100 \times 100$ : зависимости $M / \mathcal{N}$ от $h($ а) и $T \chi / \mathcal{N}$ от $h$ (б) при разных температурах $T=0.01,0.3,0.5$. Заполненые треугольники соответствуют предсказаниям модели жестких квадратов, которые справедливы только в окрестности $h_{1}$ при очень низких температурах.

3.2. Фрустрированный двойной спиновый слой. Двумерный случай более сложен, так как у нас нет аналитического решения для модели решеточного газа на квадратной решетке с отталкиванием ближайших соседей. Поэтому мы используем формулы, выражающие $M(T, h, N)$ и $\chi(T, h, N)$ через средние в большом каноническом ансамбле $\bar{n}$ и $\overline{n^{2}}$ (см. уравнения $(3.1)$ ), и вычисляем $\bar{n}$ и $\overline{n^{2}}$, используя численное моделирование по классическому методу Монте-Карло для больших систем. А именно, мы рассматриваем системы, состоящие из $\mathcal{N}=50 \times 50$ и $\mathcal{N}=100 \times 100$ узлов, используем обычный алгоритм Метрополиса и выполняем 220000 шагов Монте-Карло.

Некоторые результаты для зависимостей $M$ от $h$ и $\chi$ от $h$ при низких температурах, полученные на основе численного моделирования по классическому методу Монте-Карло $\bar{n}$ и $\overline{n^{2}}$ для модели решеточного газа, представлены на рис. 4. Температурные зависимости $M$ и $\chi$ при разных полях $h$, полученные на основе численного моделирования по классическому методу Монте-Карло для модели решеточного газа, показаны на рис. 5. Представленные результаты обсуждаются в п. 3.3.

3.3. Обсуждение результатов. Перейдем к обсуждению полученных результатов для низкотемпературных процессов намагничивания для двух рассмотренных фрустрированных квантовых антиферромагнетиков Гейзенберга. При нулевой температуре $T=0$ кривая намагничивания $M(h)$ имеет очень простой вид, а именно состоит только из плато и скачков, т.е. $M(T=0, h, N) / \mathcal{N}=1,1 / 2$ или 0 , если $h_{1}<h, h_{2}<h<h_{1}$ или $h<h_{2}$ (см. работу [6]). В результате восприимчивость $\chi(T=0, h, N)$ равна нулю в состояниях, соответствующих плато, т. е. для всех значений $h \neq h_{2}$ и $h \neq h_{1}$. Скачки $M(T=0, h, N)$ при $h=h_{2}$ и $h=h_{1}$ приводят к $\delta$-образным сингулярностям восприимчивости. Такое поведение кривой намагниченности при нулевой температуре отображает изменение основных состояний при изменении $h$, т. е. две полностью пустые подрешетки для $h_{1}<h$, одна полностью 

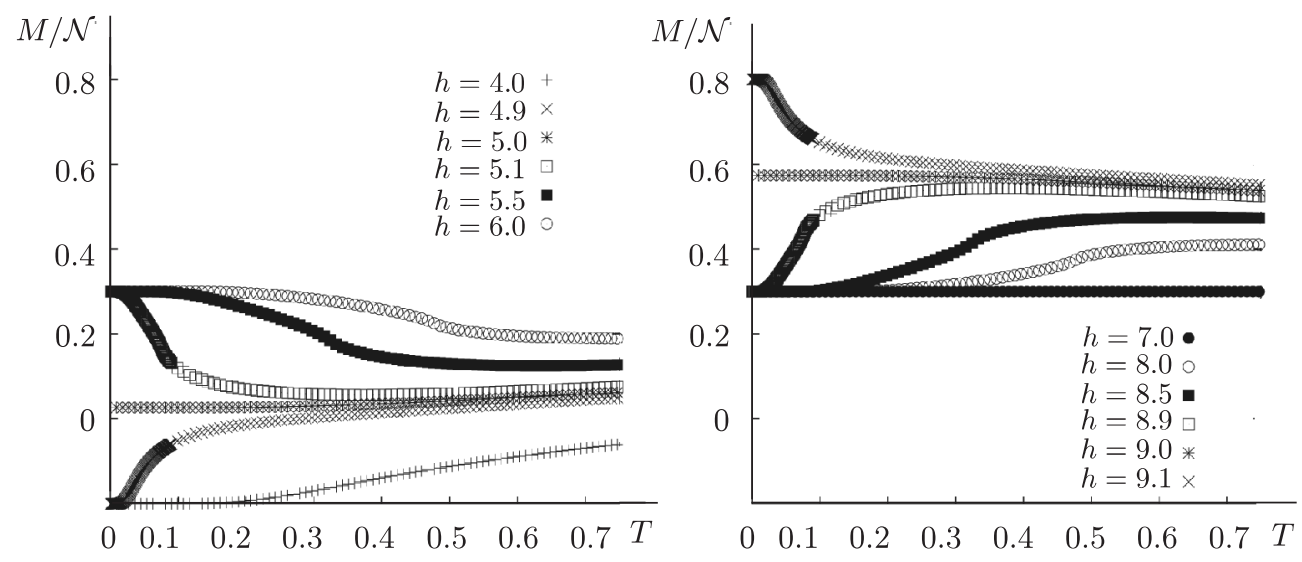

a
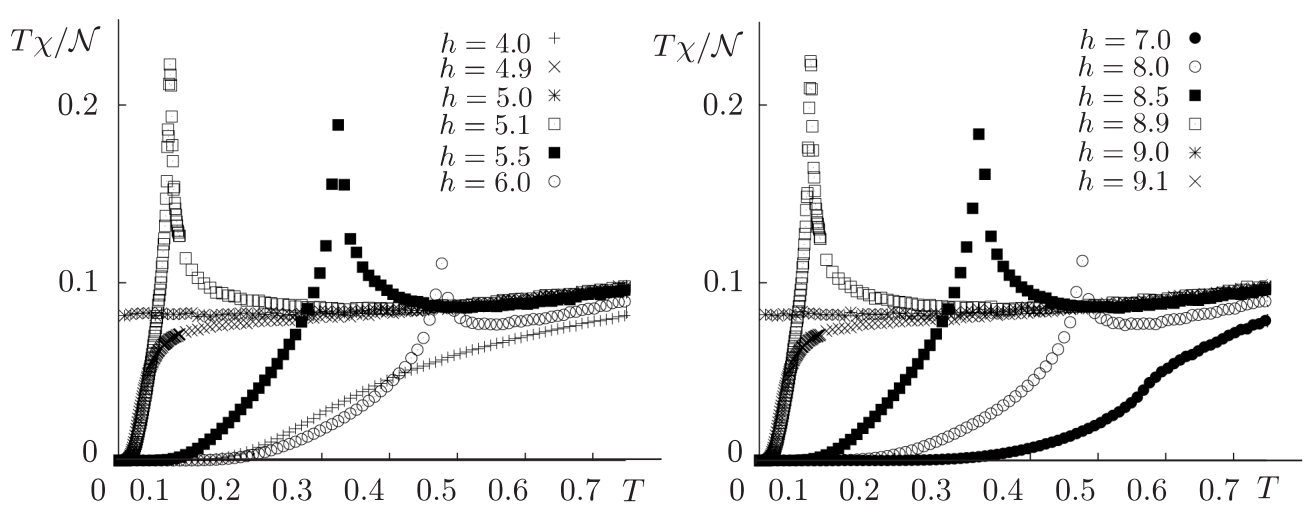

6

Рис. 5. Результаты, полученные по классическому методу Монте-Карло для фрустрированного двойного спинового слоя (2.1) с $J_{1}=1, J_{2}=5, \mathcal{N}=50 \times 50$ и $\mathcal{N}=100 \times 100$ : зависимости $M / \mathcal{N}$ от $T$ (а) и $T \chi / \mathcal{N}$ от $T$ (б) при разных полях $h=4.0, \ldots, 9.1$.

заполненная локализованными магнонами подрешетка и полностью пустая другая подрешетка для $h_{2}<h<h_{1}$ или полностью заполненные локализованными магнонами обе подрешетки для $h<h_{2}$. При $h=h_{1}$ энергия (основных) состояний с различным числом (независимых) локализованных магнонов одна и та же, что приводит к скачку кривой намагничивания. При $h=h_{2}$ энергии основных состояний в подпространствах с $S^{z}=\mathcal{N} / 2-r, r=1, \ldots, \mathcal{N} / 2$, также одинаковы, что приводит к другому скачку кривой намагничивания и $\delta$-образной сингулярности в зависимости $\chi(T=0, h, N)$ от $h$ при $h=h_{2}$.

При ненулевых температурах $T>0$ картина решеточного газа предполагает разные сценарии для одномерного и двумерного случаев. Для фрустрированной двуногой спиновой лестницы заполнение обеих подрешеток локализованными магнонами увеличивается с уменьшением $h$ (и ведет к уменьшению $M$ ), однако при конечных 
температурах обе подрешетки заполнены одинаковым числом локализованных магнонов. Плавное уменьшение $M$ с двумя крутыми участками в окрестности $h_{1}$ и $h_{2}$ ведет к двум пикам в восприимчивости $\chi$ при низких температурах (см. рис. 3 ).

Ситуация для фрустрированного двойного спинового слоя иная из-за существования упорядоченной фазы с дальним порядком при $T / J_{1}<T_{0} / J_{1}=1 /[2 \ln (\sqrt{2}+1)] \approx$ 0.567296 (см. фазовую диаграмму на рис. 2б). Для заданной температуры $T<T_{0}$ при изменении магнитного поля (что соответствует горизонтальной линии на фазовой диаграмме на рис. 2б) система претерпевает два фазовых перехода при $h_{1}(T)$ и $h_{2}(T)$, где линия $T=$ const пересекает критическую линию $T_{\mathrm{c}}(h)$. (Очевидно, что $h_{1}(T=0)=h_{1}$ и $h_{2}(T=0)=h_{2}$.) Для полей между $h_{1}(T)$ и $h_{2}(T)$ существует разница в заполнении двух подрешеток, т. е. имеет место некий “антиферромагнитный” дальний порядок локализованных магнонов. Полная плотность локализованных магнонов, которая связана с однородной намагниченностью $M$, претерпевает перегиб (см. рис. 4а), что приводит к острому пику для $\chi$ (см. рис. 4б). Бесконечный наклон для $M(h)$ при $h_{1}(T)$ и $h_{2}(T)$, приводящий к $\delta$-пикам для $\chi(h)$, имеет место при $T=0$, тогда как для конечных температур $T$ хорошо выраженные пики конечны, т. е. $\chi$ не разбегается. Альтернативный способ пересечь критическую линию - зафиксировать $h$ при $h_{2}<h<h_{1}$ и изменять температуру $T \geqslant 0$. Соответствующие профили для $M$ и $T \chi$ показаны на рис. 5. Напомним, что значение намагниченности при нулевой температуре $M(T=0, h, N) / \mathcal{N}$ равно 1 , если $h_{1}<h$; равно $1 / 2$, если $h_{2}<h<h_{1}$; равно 0 , если $h<h_{2}$. Восприимчивость $\chi$ сильно подавлена в “антиферромагнитно"-упорядоченной фазе. Это указывает на то, что состояние для плато при нулевой температуре между $h_{2}$ и $h_{1}$ сохраняется и при конечных температурах (заметим, что это утверждение не имеет места для состояний для плато при $h<h_{2}$ и $\left.h_{1}<h\right)$. Опять же, температурные профили $\chi$ указывают критическую линию $T_{\mathrm{c}}(h)$ (см. рис. 5б).

Наконец, проиллюстрируем роль взаимодействующих локализованных магнонных состояний, сравнивая с результатами, которые учитывают только независимые локализованные магнонные состояния, т. е. с полученными при помощи решеточных моделей с бесконечным отталкиванием ближайших соседей (одномерная модель жестких димеров и двумерная модель жестких квадратов [3], [4]). Соответствующие данные показаны на рис. 3 и 4 заполненными треугольниками. Предсказания моделей жестких димеров и жестких квадратов правильны при низких температурах и при $h$ в окрестности $h_{1}$, когда независимые локализованные магнонные состояния действительно доминируют в статистической сумме рассматриваемых фрустрированных квантовых спиновых систем. Понятно, что учет взаимодействующих локализованных магнонных состояний приводит к значительно большей области, в которой эффективное описание справедливо, по сравнению с описанием с помощью жестких объектов.

\section{4. ВЫВОДЫ}

Мы рассмотрели низкотемпературные процессы намагничивания для двух фрустрированных квантовых антиферромагнетиков Гейзенберга. Возможность детального исследования этих систем связана с возникновением степеней свободы Изинга в режиме сильного взаимодействия. В результате фрустрированные квантовые системы оказались подобными классическим моделям решеточного газа или, эквивалентно, антиферромагнетикам Изинга в однородном магнитном поле. Классические 
решеточные системы значительно легче изучать либо аналитически (в одномерном случае), либо с использованием численного моделирования по классическому методу Монте-Карло (в двумерном случае). Наиболее интересные черты низкотемпературных процессов намагничивания связаны с фазовым переходом порядок-беспорядок при ненулевой температуре, который имеет место в двумерном случае. Мы показали, что полевые и температурные зависимости однородных намагниченности и восприимчивости сигнализируют об упорядочении локализованных магнонов на вертикальных связях фрустрированного двойного спинового слоя. Прыжки и плато, найденные при нулевой температуре в обеих системах, типичны для сильно фрустрированных квантовых магнетиков [1]. Прыжки размываются даже при довольно низких температурах, когда плато все еще хорошо видно. Характерной чертой при конечных температурах являются острые пики для $\chi(T, h, N)=\partial M(T, h, N) / \partial h$ на концах плато, которые имеют место для спиновой лестницы. Такие пики были найдены теоретически и экспериментально, например, для спин-1/2 антиферромагнетика Гейзенберга на треугольной решетке [15], [16] и для фрустрированных магнитных молекул [17]. Для модели двойного спинового слоя острые пики для $\chi(T, h, N)$ связаны с фазовым переходом, который имеет место в этой системе. В результате они появляются при $T<T_{0}=J_{1} /[2 \ln (\sqrt{2}+1)] \approx 0.567296 J_{1}$, т. е. вплоть до довольно больших температур.

Хотя в настоящей статье мы ограничили рассмотрение случаем двух конкретных фрустрированных решеток, его можно распространить на другие решетки, например на фрустрированную трехногую спиновую лестницу в одномерном случае [6], [18] или на фрустрированные двойные спиновые слои, состоящие из двух треугольных, шестиугольных и др. спиновых слоев в двумерном случае.

Более общо, принимая во внимание режим сильного взаимодействия, для которого справедливо наше рассмотрение, мы можем говорить о системе слабо взаимодействующих димеров, организованных в различные решетки разных пространственных измерений таким образом, что синглетные состояния на димерах являются локализованными. Для достижения этой цели требуются фрустрированные взаимодействия. С экспериментальной точки зрения (о твердотельных реализациях некоторых подобных моделей см. работы [19], [20]) было бы интересно рассмотреть случай, когда условия локализации несколько нарушены (см., например, работу [21], в которой обсуждаются кривые намагниченности для такой фрустрированной двуногой спиновой лестницы при нулевой температуре).

Благодарности. Один из авторов (О. Держко) признателен DFG за финансовую поддержку и благодарен Университету Магдебурга за гостепреимство в конце 2010 г., когда была выполнена основная часть исследований.

\section{Список литературы}

[1] A. Honecker, J. Phys.: Condens. Matter, 11:24 (1999), 4697-4713, arXiv: cond-mat/9902163; A. Honecker, J. Schulenburg, J. Richter, J. Phys.: Condens. Matter, 16:11 (2004), S749-S758, arXiv: cond-mat/0309425; J. Richter, J. Schulenburg, A. Honecker, "Quantum magnetism in two dimensions: From semi-classical Néel order to magnetic disorder", Quantum Magnetism, Lecture Notes in Physics, 645, eds. U. Schollwöck, J. Richter, D. J. J. Farnell, R. F. Bishop, Springer, Berlin, 2004, 85-153.

[2] J. Schnack, H.-J. Schmidt, J. Richter, J. Schulenburg, Eur. Phys. J. B, 24:4 (2001), 475-481, arXiv: cond-mat/0108432; J. Schulenburg, A. Honecker, J. Schnack, J. Richter, 
H.-J. Schmidt, Phys. Rev. Lett., 88:16 (2002), 167207, 4 pp., arXiv: cond-mat/0108498; J. Richter, J. Schulenburg, A. Honecker, J. Schnack, H.-J. Schmidt, J. Phys.: Condens. Matter, 16:11 (2004), S779-S784; J. Richter, ФHT, 31:8-9 (2005), 918-928; O. Derzhko, J. Richter, A. Honecker, H.-J. Schmidt, ФHT, 33:9 (2007), 982-996, arXiv: cond-mat/0612281; J. Richter, O. Derzhko, "Correlated systems on geometrically frustrated lattices: from magnons to electrons", Condensed Matter Physics in the Prime of the 21st Century. Phenomena, Materials, Ideas, Methods, 43rd Karpacz Winter School of Theoretical Physics (Lạdek Zdrój, Poland, 5-11 February 2007), ed. J. Jȩdrzejewski, World Scientific, Singapore, 2008, 237-270.

[3] M. E. Zhitomirsky, H. Tsunetsugu, Phys. Rev. B, 70:10 (2004), 100403(R), 4 pp., arXiv: cond-mat/0405578; O. Derzhko, J. Richter, Phys. Rev. B, 70:10 (2004), 104415, 6 pp., arXiv: cond-mat/0404204; M. E. Zhitomirsky, H. Tsunetsugu, Progr. Theor. Phys. Suppl., 2005, № 160, 361-382, arXiv: cond-mat/0506327; O. Derzhko, J. Richter, Eur. Phys. J. B, 52:1 (2006), 23-36, arXiv: cond-mat/0604023.

[4] J. Richter, O. Derzhko, T. Krokhmalskii, Phys. Rev. B, 74:14 (2006), 144430, 5 pp., arXiv: cond-mat/0606806; O. Derzhko, J. Richter, T. Krokhmalskii, Acta Phys. Polon. A, 113:1 (2008), 433-436.

[5] O. Derzhko, T. Krokhmalskii, J. Richter, Phys. Rev. B, 82:21 (2010), 214412, 14 pp., arXiv: 1009.3828.

[6] A. Honecker, F. Mila, M. Troyer, Eur. Phys. J. B, 15:2 (2000), 227-233, arXiv: cond-mat/9910438.

[7] P. Chen, C.-Y. Lai, M.-F. Yang, Phys. Rev. B, 81:2 (2010), 020409(R), 4 pp., arXiv: 0910.5081 .

[8] H.-J. Schmidt, J. Phys. A, 35:31 (2002), 6545-6555, arXiv: cond-mat/0203270.

[9] H.-J. Schmidt, J. Richter, R. Moessner, J. Phys. A, 39:34 (2006), 10673-10690, arXiv: cond-mat/0604649.

[10] E. Müller-Hartmann, J. Zittartz, Z. Phys. B, 27:3 (1977), 261-266.

[11] X. N. Wu, F. Y. Wu, Phys. Lett. A, 144:3 (1990), 123-126.

[12] X.-Z. Wang, J. S. Kim, Phys. Rev. Lett., 78:3 (1997), 413-416.

[13] S. J. Penney, V. K. Cumyn, D. D. Betts, Physica A, 330:3-4 (2003), 507-518.

[14] Р. Бэкстер, Точно решаемые модели в статистической механике, Мир, М., 1985.

[15] D. J. J. Farnell, R. Zinke, J. Schulenburg, J. Richter, J. Phys.: Condens. Matter, 21:40 (2009), 406002, 12 pp., arXiv: 0908.2881.

[16] T. Ono, H. Tanaka, H. Aruga Katori, F. Ishikawa, H. Mitamura, T. Goto, Phys. Rev. B, 67:10 (2003), 104431, 7 pp.

[17] C. Schröder, H. Nojiri, J. Schnack, P. Hage, M. Luban, P. Kögerler, Phys. Rev. Lett., 94:1 (2005), 017205, 4 pp.

[18] M. Maksymenko, O. Derzhko, J. Richter, Acta Phys. Polon. A, 119:6 (2011), 860-862; Low-temperature properties of the quantum Heisenberg antiferromagnet on some one-dimensional lattices containing equilateral triangles, preprint ICMP-10-08E, Inst. Condens. Matter Phys., Lvov, 2010, http://www.icmp.lviv.ua/preprints/2010.html.

[19] N. B. Ivanov, Cond. Matter Phys., 12:3 (2009), 435-447, arXiv: 0909.2182.

[20] G. Seeber, P. Kögerler, B. M. Kariuki, L. Cronin, Chem. Commun., 2004:11 (2004), 1580-1581; N. B. Ivanov, J. Schnack, R. Schnalle, J. Richter, P. Kögerler, G. N. Newton, L. Cronin, Y. Oshima, H. Nojiri, Phys. Rev. Lett., 105:3 (2010), 037206, 4 pp., arXiv: 1004.2373.

[21] J.-B. Fouet, F. Mila, D. Clarke, H. Youk, O. Tchernyshyov, P. Fendley, R. M. Noack, Phys. Rev. B, 73:21 (2006), 214405, 13 pp., arXiv: cond-mat/0603609. 